\title{
Characteristics of gastrointestinal hemorrhage associated with pancreatic cancer: A retrospective review of 246 cases
}

\author{
YU WANG $^{1}$, CAIJUN YUAN ${ }^{2}$ and XIAOMEI LIU ${ }^{2}$ \\ ${ }^{1}$ Department of Oncology, Graduate School of Liaoning Medical University; ${ }^{2}$ Department of Oncology, \\ The First Affiliated Hospital of Liaoning Medical University, Jinzhou, Liaoning 121000, P.R. China
}

Received December 16, 2014; Accepted March 4, 2015

DOI: $10.3892 / \mathrm{mco} .2015 .563$

\begin{abstract}
While gastrointestinal (GI) hemorrhage is common in the general population, few studies have evaluated large numbers of GI hemorrhage patients with pancreatic cancer. The clinical features and potential risk factors of GI hemorrhage with pancreatic cancer was investigated in the present study and the effect of GI hemorrhage on survival rate was examined. Patients enrolled in the present study had pathologically proven pancreatic cancer, and received treatment between August 2006 and 2012. Their medical records were retrospectively reviewed. The data for the present study were obtained from a review of 246 patients with pancreatic cancer (average age, 63.4 \pm 10.92 years; 190 male cases, 56 female cases). In addition, 73 cases had stage I-II, 173 had stage III-IV, and only 67 cases (27.2\%) were candidates for curative pancreatectomy. Among them, 32 cases (13.0\%) were clinically diagnosed with GI hemorrhage. A total of 24 cases were male patients and the other 8 cases were female, the cases of hemorrhage history and alcoholism were 2 and 29 cases, respectively. The major initial clinical symptoms of GI hemorrhage included 18 patients with melena or blood stool (56.25\%), 9 with haematemesis $(28.13 \%)$, 3 with abdominal distention $(9.37 \%)$ and 2 with stomach ache $(6.25 \%)$. The independent risk factor for GI hemorrhage was tumor initial stage of IV. A continuous increase in carbohydrate antigen 19-9 (CA19-9) may be a warning of GI hemorrhage, particularly when it is $>1,000 \mathrm{U} / \mathrm{ml}$. The most frequent method of hemostasis was combination therapy $(n=12,37.5 \%)$. Only 3 cases $(9.3 \%)$ of these 32 GI hemorrhage patients were blood stanched and only 10 patients $(31.2 \%)$ received gastroscopy. The time from GI hemorrhage to fatality is extremely short (median 30 days, range from $1 \mathrm{~h}$ to 65 days),
\end{abstract}

Correspondence to: Professor Caijun Yuan, Department of Oncology, The First Affiliated Hospital of Liaoning Medical University, 2 Renmin Street, Section 5, Jinzhou, Liaoning 121000, P.R. China

E-mail: caijunyunyuan521@163.com

Key words: gastrointestinal hemorrhage, pancreatic cancer, risk factor, overall survival and the median overall survival time of the patients with GI hemorrhage was 9.0 months (range, 2.0-16.0 months) and was significantly shorter than that of patients without GI hemorrhage [14.5 months (range, 0.5-48.0 months)]. In conclusion, although GI hemorrhage was not common in patients with pancreatic cancer, it is critical. GI hemorrhage was controlled with endoscopic hemostasis. Clinicians should fully assess the risk factors of GI hemorrhage (such as alcohol, smoking, past hemorrhage history, initial stage, tumor location and CA19-9 level at diagnosis of pancreatic cancer) when the pancreatic cancer patients were on admission, particularly for patients of the late stage, preventive measures should be investigated to reduce suffering.

\section{Introduction}

Pancreatic cancer is the fourth leading cause of cancer-related fatalities in the USA with a 5-year survival rate of 4-6\% $(1,2)$. The only curative treatment for pancreatic cancer is surgical resection (3). However, $25 \%$ of patients with pancreatic cancer are candidates for curative pancreatectomy. In recent years, chemoradiotherapy (CRT) has been considered as a reasonable treatment for locally advanced pancreatic cancer (LAPC). The main complications of pancreatic cancer include diabetes, thrombophlebitis, weight loss and mental symptoms, while GI hemorrhage with serious prognosis is so unusual that investigators seldom have the research and systematic exposition, leading to a long-term limit in the research in this field.

Gastrointestinal (GI) hemorrhage is caused by numerous different lesions and varies greatly in severity, ranging from clinically insignificant to life threatening (4). It is a frequent cause of hospitalization, although a number of advances have been made in diagnosis and treatments. Studies from the United States indicated that hospitalizations for upper GI (UGI) hemorrhage in the early 1990s occurred at an annual incidence of $\sim 100 / 100,000$ population and were $\sim 5$ times more common than hospitalizations for lower GI hemorrhage (5-9). UGI hemorrhage usually causes fatalities in 6-14\% of those it affects $(9,10)$. Patient morbidity and mortality is typically proportional to the degree of initial blood loss, the rate of rebleeding following endoscopy, underlying illnesses, and of note, the age of the patient age (10-13). A systematic study by Laine et al (14) suggested that the most common causes of UGI hemorrhage include peptic ulcer disease 
[32.1/100,000, (with gastric ulcers more than duodenal)], gastritis or duodenitis $(10.0 / 100,000)$, angiodysplasia (5.02/100,000), esophageal ulcer $(2.71 / 100,000)$, esophageal varices (1.25/100,000), Dieulafoy's lesion (1.26/100,000), gastrojejunal ulcer $(1.49 / 100,000)$ and unspecified peptic ulcer $(0.83 / 100,000)$. Abdominal arteriography may localize GI hemorrhage sources in approximately one-third of cases. Selective embolization may provide definitive hemostasis in the outcomes of the majority of instances of GI hemorrhage (15). However, there are few studies on the rate and the risk factors of GI hemorrhage in patients with pancreatic cancer, which lead to an ambiguous GI hemorrhage rate of pancreatic cancer and controversy remains in the cause, treatment and outcome.

Therefore, the present study evaluated the incidence and survival time of the GI hemorrhage patients in pancreatic cancer in The First Affiliated Hospital of Liaoning Medical University (Jinzhou, China) over the past 6 years, to assess the clinical characteristics and to determine the risk factors for it. The study aimed to overcome the incomplete estimates of GI hemorrhage patients in pancreatic cancer in the previous studies.

\section{Materials and methods}

Patients. A total of 246 pancreatic cancer patients (male:female, 190:56; median age, 63.3 years) admitted at The First Affiliated Hospital of Liaoning Medical University between August 2006 and 2012 were retrospectively evaluated. Pancreatic cancer was defined according to the National Comprehensive Cancer Network (NCCN; http://www.nccn. org/professionals/physician_gls/f_guidelines.asp) for oncologists. Inclusion criteria included pathologically-proven pancreatic adenocarcinoma, $>18$ years and the Eastern Cooperative Oncology Group performance status of 0 to 2 . Exclusion criteria included patients who did not have pathologically-proven pancreatic adenocarcinoma. Patients with psychosis, sepsis (the presence of $\geq 2$ of the following features was considered evidence of sepsis: i) Temperature $38^{\circ} \mathrm{C}$ or $36^{\circ} \mathrm{C}$, ii) heart rate 90 beats $/ \mathrm{min}$, iii) respiratory rate 20 breaths/min or iv) leukocyte cell count 12,000 or $4,000 / \mathrm{mm}^{3}$ ), $10 \%$ band forms (16) or coagulopathy (coagulopathy was defined as a platelet count of $50,000 / \mathrm{mm}^{3}$, a prothrombin time/international normalized ratio of 1.5 or an activated partial thromboplastin time 2.03 the control value) were also excluded for the protocol analysis. The study was approved by the Ethics Committee of the First Affiliated Hospital of Liaoning Medical University.

GI hemorrhage. GI hemorrhage was defined as any episode of fresh blood or coffee ground materials in nasogastric aspirate, hematemesis, melena or bloody stool. GI hemorrhage was considered severe if accompanied by hypotension (systolic blood pressure, $100 \mathrm{mmHg}$ ), a decrease in the hemoglobin (Hb) level of $2 \mathrm{~g} / \mathrm{dl}$ or requiring blood transfusion (17). In the study, to determine the risk factors for GI hemorrhage, the history of peptic ulcer, alcohol, smoking or hemorrhage were recorded. The treatment for GI hemorrhage includes endoscopic hemostasis, angiography, embolization and conservative care. The methods of endoscopic hemostasis were hypertonic saline-epinephrine injection, human plasmin thrombin injection, argon plasma coagulation or hemoclipping. Rebleeding was confined to episodes within 7 days after successful hemostasis therapy.

Treatment for pancreatic cancer. For regression analysis, regimens of treatment methods for pancreatic cancer mainly included surgery, chemotherapy and CRT. The specific scheme is formulated in accordance with the NCCN guidelines. All the chemotherapy regimens of CRT performed for LAPC were classified into three groups: Gemcitabine, 5-fluorouracil (5-FU) and 5-FU plus gemcitabine. The gemcitabine group was administered $1,000 \mathrm{mg} / \mathrm{m}^{2}$ of gemcitabine on days 1,8 and 15 of a 4 -week regimen or gemcitabine (same as above) along with $70 \mathrm{mg} / \mathrm{m}^{2}$ of cisplatin on day 1 of the regimen. The 5-FU group received either $5-\mathrm{FU}\left(1,000 \mathrm{mg} / \mathrm{m}^{2}\right.$ on days 1-3 of a 4-week regimen) or TS-1 (60-80 mg for 2 weeks), or a combination of $5-\mathrm{FU}\left(1,000 \mathrm{mg} / \mathrm{m}^{2}\right.$ on days $\left.1-3\right)$, etoposide $\left(100 \mathrm{mg} / \mathrm{m}^{2}\right.$ on days $\left.1-3\right)$ and cisplatin $\left(70 \mathrm{mg} / \mathrm{m}^{2}\right.$ on day 1). For the 5-FU plus gemcitabine group, $1,000 \mathrm{mg} / \mathrm{m}^{2}$ of 5-FU was administered on days $1-3$ and $1,000 \mathrm{mg} / \mathrm{m}^{2}$ gemcitabine on days 1,8 and 15 of a 4 -week regimen. All the radiotherapy regimens of CRT performed for pancreatic cancer were either three-dimensional conformal radiotherapy (total dose, 4,000-5,400 cGy; one dose, 180-250 cGy; fraction, 28) or intensity-modulated radiotherapy (total dose, 4,200-6,000 cGy; one dose, 200-293 cGy; fraction, 25).

Statistical analysis. To investigate the baseline characteristics, the $\chi^{2}$ test and Student's t-test were used. Categorical variables were analyzed by the $\chi^{2}$ test and continuous variables were assessed by the Student's t-test. To evaluate the survival effect of GI hemorrhage, Cox regression test was used. The Kaplan-Meier method and the log-rank test were used to compare the survival rate between patients with and without GI hemorrhage. Univariate analysis and multivariate logistic regression were used to detect independent risk factors associated with hemorrhage for pancreatic cancer and prognosis. $\mathrm{P}<0.05$ was considered to indicate a statistically significant difference. Analyses were performed using SPSS software, version 17.0 (SPSS Inc., Chicago, IL, USA).

\section{Results}

Patient characteristics. During the 6-year study period, 246 patients with pancreatic cancer were eligible for analysis (Table I). The average age at the time of the diagnosis of pancreatic cancer was $63.4 \pm 10.92$ years. Male patients accounted for $77.24 \%$ of the population, $\sim 2$ times more than females. A total of 3 patients suffered from a history of hemorrhage prior to the diagnosis of pancreatic cancer. The tumors were mostly located at the pancreatic head $(57.72 \%)$. The most common symptom when diagnosed with pancreatic cancer is abdominal distension or pain $(53.66 \%)$. The level of carbohydrate antigen 19-9 (CA19-9) was 549.1+967.1 U/ml. Surgery was performed for 67 patients (27.25\%) and CRT was delivered to 77 patients $(31.30 \%)$, with the specific dose determined according to the NCCN guidelines for pancreatic cancer (Fig. 1). The median follow-up period was 14.6 months (range, 1.5-60.1 months). 
Table I. Baseline clinical characteristics of pancreatic cancer patients.

\begin{tabular}{|c|c|}
\hline Variables & Number \\
\hline Gender, male/female & $190 / 56$ \\
\hline Age, years ${ }^{\mathrm{a}}$ & $63.4 \pm 10.92$ \\
\hline Alcohol, yes/no & $167 / 79$ \\
\hline Smoking, yes/no & $140 / 106$ \\
\hline Past hemorrhage history, yes/no & $3 / 243$ \\
\hline Past ulcer history, yes/no & $22 / 224$ \\
\hline Past diabetes history, yes/no & $25 / 221$ \\
\hline Past cirrhosis history, yes/no & $30 / 216$ \\
\hline Initial stage, I/II/III/IV & $67 / 6 / 29 / 144$ \\
\hline $\begin{array}{l}\text { Recived treatment prior to hemorrhage, } \\
\text { chemotherapy/concurrent } \\
\text { chemoradiotherapy/others }\end{array}$ & $156 / 60 / 30$ \\
\hline $\begin{array}{l}\text { Initial clinical symptoms, } \\
\text { stained yellow/abdominal distension } \\
\text { or pain/diabete/ileus/others }\end{array}$ & $64 / 132 / 13 / 13 / 24$ \\
\hline $\begin{array}{l}\text { Tumor location, } \\
\text { head of pancreas/body of pancreas/ } \\
\text { tail of pancreas }\end{array}$ & $142 / 49 / 55$ \\
\hline CA19-9 at diagnosis, $\mathrm{U} / \mathrm{ml}$ & $549.1 \pm 967.1$ \\
\hline \multicolumn{2}{|l|}{ Initial blood pressure } \\
\hline Systolic, $\mathrm{mmHg}^{\mathrm{a}}$ & $138.1 \pm 17.2$ \\
\hline Diastolic, $\mathrm{mmHg}^{\mathrm{a}}$ & $82.7 \pm 11.3$ \\
\hline Initial heart rates ${ }^{\mathrm{a}}$ & $92.0 \pm 20.1$ \\
\hline Hemoglobin, $\mathrm{g} / \mathrm{dl}^{\mathrm{a}}$ & $138.3 \pm 24.7$ \\
\hline Platelet, $10^{9} / 1^{\text {a }}$ & $239.0 \pm 67.2$ \\
\hline INR/PTT, $\sec ^{\mathrm{a}}$ & $1.21 \pm 14.7 / 46.3 \pm 41.6$ \\
\hline
\end{tabular}

${ }^{a}$ Mean \pm standard deviation. INR, international normalized ratio; PTT, partial thromboplastin time; Hb, hemoglobin; GI, gastrointestinal; CA19-9, carbohydrate antigen 19-9.

GI hemorrhage. There were 32 patients (13.0\%) who suffered from GI hemorrhage (Table II). The initial $\mathrm{Hb}$ was $11.2 \pm 25.5 \mathrm{~g} / \mathrm{dl}$, which decreased to $8.4 \pm 17.1 \mathrm{~g} / \mathrm{dl}$ when bleeding and the median CA19-9 level increased from $456.7 \pm 547.1$ to $1,408.5 \pm 789.4$. Among the total $32 \mathrm{GI}$ hemorrhage patients, there were 7 patients belonging to the mild GI hemorrhage group with grade 1,15 patients in the moderate GI hemorrhage group with grade 2 or 3 and 10 patients in the severe GI hemorrhage group with grade 4 or 5 . Due to a bad physical state, conservative care was delivered to 11 cases and endoscopic hemostasis to 20 cases, while only 1 case underwent angiography and embolization. Prior to GI hemorrhage, surgical treatment was delivered to 5 patients $(15.6 \%)$ and CRT was delivered to 25 patients (78.13\%). The most common major initial clinical symptoms for GI hemorrhage were melena or blood stool and haematemesis. Due to a bad physical state, only 20 patients underwent UGI endoscopy. The results showed the cause
Table II. Clinical characteristics of pancreatic cancer patients with GI hemorrhage $(n=32)$.

\begin{tabular}{|c|c|}
\hline Variables & Number \\
\hline Gender, male/female & $24 / 8$ \\
\hline Age, years ${ }^{\mathrm{a}}$ & $62.4 \pm 8.7$ \\
\hline Alcohol, yes/no & $29 / 3$ \\
\hline Smoking, yes/no & $25 / 7$ \\
\hline Past hemorrhage history, yes/no & $2 / 30$ \\
\hline \multicolumn{2}{|l|}{ Hemoglobin, g/dl } \\
\hline Initial & $11.2 \pm 25.5$ \\
\hline At bleeding & $8.4 \pm 17.1$ \\
\hline \multicolumn{2}{|l|}{ CA19-9 level, U/ml } \\
\hline Initial & $456.7 \pm 547.1$ \\
\hline At bleeding & $1,408.5 \pm 789.4$ \\
\hline \multicolumn{2}{|l|}{ Severity } \\
\hline Mild & 7 \\
\hline Moderate & 15 \\
\hline Severe & 10 \\
\hline \multicolumn{2}{|l|}{ Treatment } \\
\hline Endoscopic hemostasis & 20 \\
\hline Angiography and embolization & 1 \\
\hline Conservative care & 11 \\
\hline $\begin{array}{l}\text { Initial clinical symptoms at diagnosis } \\
\text { of pancreatic cancer, } \\
\text { stained yellow/abdominal distension } \\
\text { or pain/diabete/ileus/others }\end{array}$ & $12 / 18 / 0 / 1 / 1$ \\
\hline Time to mortality from hemorrhage & $31.5 \pm 21.6$ days \\
\hline Survival time from diagnosis & $10.0 \pm 6.2$ months \\
\hline H. pylori infection, yes/no & $10 / 22$ \\
\hline Hemorrhage related mortality, yes/no & $20 / 12$ \\
\hline \multicolumn{2}{|c|}{ Initial clinical symptoms of GI hemorrhage } \\
\hline Melena or blood stool & 18 \\
\hline Haematemesis & 9 \\
\hline Abdominal distention & 3 \\
\hline Stomach ache & 2 \\
\hline
\end{tabular}

${ }^{a}$ Mean \pm standard deviation. GI, gastrointestinal; H. pylori, Helicobacter pylori; CA19-9, carbohydrate antigen 19-9.

of bleeding to be a gastric ulcer in 8 patients, duodenal ulcer in 5, radiation gastritis in 3 and another reason in 4 . UGI endoscopy was not performed in 12 patients upon the rejection of their guardians or a bad physical state. As the patients were in terminal stages, the guardians did not want them to undergo any more examinations. Hemorrhage was successfully stopped by endoscopic treatment in 12 patients (37.5\%). The methods of endoscopic hemostasis included hypertonic saline-epinephrine injection, argon plasma coagulation, human plasmin thrombin injection or hemoclipping. Embolization was performed in 1 patient and hemorrhage was finally stopped. However, 7 cases rebled 
Table III. Baseline clinical characteristics of pancreatic cancer patients.

\begin{tabular}{|c|c|c|}
\hline \multirow[b]{2}{*}{ Variables } & \multicolumn{2}{|l|}{ GI hemorrhage } \\
\hline & Presence, n (\%) & P-value \\
\hline Gender, male/female & $24(12.6) / 8(14.3)$ & NS \\
\hline \multicolumn{3}{|l|}{ Age, years ${ }^{\mathrm{a}}$} \\
\hline$\leq 65$ & $22(12.4)$ & NS \\
\hline$>65$ & $10(14.7)$ & \\
\hline Alcohol, yes/no & $29(17.4) / 3(3.8)$ & $<0.01$ \\
\hline Smoking, yes/no & $25(17.9) / 7(6.6)$ & $<0.01$ \\
\hline Past hemorrhage history, yes/no & $2(66.7) / 30(12.3)$ & $<0.01$ \\
\hline Past ulcer history, yes/no & $3(13.6) / 29(12.9)$ & NS \\
\hline Past diabetes history, yes/no & $4(16.0) / 28(12.7)$ & NS \\
\hline Past cirrhosis history, yes/no & $2(6.7) / 30(13.9)$ & NS \\
\hline Initial stage, I/II/III/IV & $8(11.9) / 4(66.7) / 6(20.7) / 14(9.7)$ & $<0.01$ \\
\hline $\begin{array}{l}\text { Recived treatment prior to hemorrhage, } \\
\text { chemotherapy/concurrent chemoradiotherapy/others }\end{array}$ & $18(11.5) / 8(13.3) / 6(20.0)$ & NS \\
\hline $\begin{array}{l}\text { Tumor location, } \\
\text { head of pancreas/body of pancreas/tail of panaceas }\end{array}$ & $13(9.2) / 13(26.5) / 6(10.9)$ & $<0.01$ \\
\hline $\begin{array}{l}\text { Initial clinical symptoms, } \\
\text { stained yellow/abdominal distension or pain/diabete/ileus/others }\end{array}$ & $12(18.8) / 18(13.6) / 0(0) / 1(7.7) / 1(4.2)$ & NS \\
\hline \multicolumn{3}{|l|}{ CA19-9 at diagnosis, $\mathrm{U} / \mathrm{ml}$} \\
\hline$\leq 1,000$ & $13(7.6)$ & $<0.01$ \\
\hline$>1,000$ & $19(25.3)$ & \\
\hline \multicolumn{3}{|l|}{ Initial blood pressure } \\
\hline Systolic, $\mathrm{mmHg}^{\mathrm{a}}$ & $131.6 \pm 39.0$ & NS \\
\hline Diastolic, $\mathrm{mmHg}^{\mathrm{a}}$ & $76.0 \pm 17.5$ & NS \\
\hline Initial heart rates ${ }^{\mathrm{a}}$ & $93.0 \pm 26.5$ & NS \\
\hline Hemoglobin, $\mathrm{g} / \mathrm{dl}^{\mathrm{a}}$ & $8.4 \pm 17.1$ & NS \\
\hline Platelet, $10^{9} / 1^{\mathrm{a}}$ & $208 \pm 147$ & NS \\
\hline INR/PTT, $\mathrm{sec}^{\mathrm{a}}$ & $1.13 \pm 0.36 / 45.1 \pm 54.7$ & NS \\
\hline
\end{tabular}

${ }^{\mathrm{a}}$ Mean \pm standard deviation. NS, not significant; INR, international normalized ratio; PTT, partial thromboplastin time; Hb, hemoglobin; GI, gastrointestinal; CA19-9, carbohydrate antigen 19-9. GI, gastrointestinal.

and 18 patients in total succumbed to GI hemorrhage. The average time from GI hemorrhage to mortality was $31.5 \pm 21.6$ days and the average overall survival rate was $10.0 \pm 6.2$ months.

Risk factors for GI hemorrhage. The incidence of GI hemorrhage was $13.0(n=32)$ and $10.2 \%(n=25)$ of patients succumbed due to bleeding. The present study analyzed the association between clinical parameters and the risk of GI hemorrhage. In the univariate analysis, there was no statistically significant difference in gender, age, past ulcer history, past diabetes history, past cirrhosis history, the type of received treatment prior to hemorrhage, initial blood pressure, initial heart rates, $\mathrm{Hb}$, platelet and international normalized ratio/partial thromboplastin time between the GI hemorrhage and the no-GI hemorrhage groups (Table III). Alcohol, smoking, past hemorrhage history, initial stage, tumor location and CA19-9 level at diagnosis of pancreatic cancer $(\mathrm{P}<0.05)$ were risk factors for GI hemorrhage. Alcohol consumption was noted for 29/32 (90.6\%) patients in the GI hemorrhage group and 138/214 $(64.5 \%)$ patients in the no-GI hemorrhage group $(\mathrm{P}<0.05)$. Additionally, smoking was reported by $25 / 32$ (78.1\%) patients in the GI hemorrhage group and 115/214 (53.7\%) patients in the no-GI hemorrhage group $(\mathrm{P}<0.05)$. The factors of past hemorrhage history, initial stage at diagnosis of pancreatic cancer, tumor location and ulcer sizes and CA19-9 level at diagnosis of pancreatic cancer were significantly different between the GI hemorrhage and the no-GI hemorrhage groups $(\mathrm{P}<0.05)$.

In multivariate analysis, only initial stage IV tumors [odds ratio (OR), 21.94; 95\% confidence interval (CI), 2.274-211.642] were a significant risk factor for GI hemorrhage (Table IV). The hazard ratio was 1.246 (95\% CI, 0.754-2.060) for the effects of GI hemorrhage on the survival rate, but it was not significant (Table V). 
Table IV. Logistic multivariate regression analysis of GI hemorrhage in all patients $(\mathrm{n}=246)$.

\begin{tabular}{lccccccc}
\hline Variables & $\mathrm{B}$ & $\mathrm{SE}$ & Wald & df & P-value & Exp (B) & 95\% CI for Exp (B) \\
\hline Alcohol & -0.728 & 0.876 & 0.691 & 1 & 0.406 & 0.483 & $0.087-2.689$ \\
Smoking & 0.192 & 0.673 & 0.081 & 1 & 0.776 & 1.211 & $0.324-4.525$ \\
PHH & -22.598 & $3,991.455$ & 0.000 & 1 & 0.995 & 0.000 & 0.000 \\
Stage & & & 7.165 & 3 & 0.067 & & $0.435-3.066$ \\
Stage (1) & 0.144 & 0.498 & 0.084 & 1 & 0.772 & 1.155 & 0.000 \\
Stage (2) & -19.245 & $16,326.303$ & 0.000 & 1 & 0.999 & 0.000 & $2.274-211.642$ \\
Stage (3) & 3.088 & 1.156 & 7.132 & 1 & 0.008 & 21.940 & 0.000 \\
Tl & & & 8.250 & 2 & 0.016 & & 0.000 \\
Tl (1) & 36.977 & $5,793.616$ & 0.000 & 1 & 0.995 & $1.146 \mathrm{E} 16$ & $0.172-1.239$ \\
Tl (2) & 32.788 & $5,793.616$ & 0.000 & 1 & 0.995 & $1.737 \mathrm{E} 14$ & 0.462 \\
CA19-9 & -0.772 & 0.503 & 2.353 & 1 & 0.125 & 0.000 & \\
Constant & -15.521 & $4,199.319$ & 0.000 & 1 & 0.997 & 0.000 \\
\hline
\end{tabular}

GI, gastrointestinal; SE, standard error; CI, confidence interval; CA19-9, carbohydrate antigen 19-9; B, regression coefficient; T, tumor; PHH, primary human hepatocytes; df, degrees of freedom.

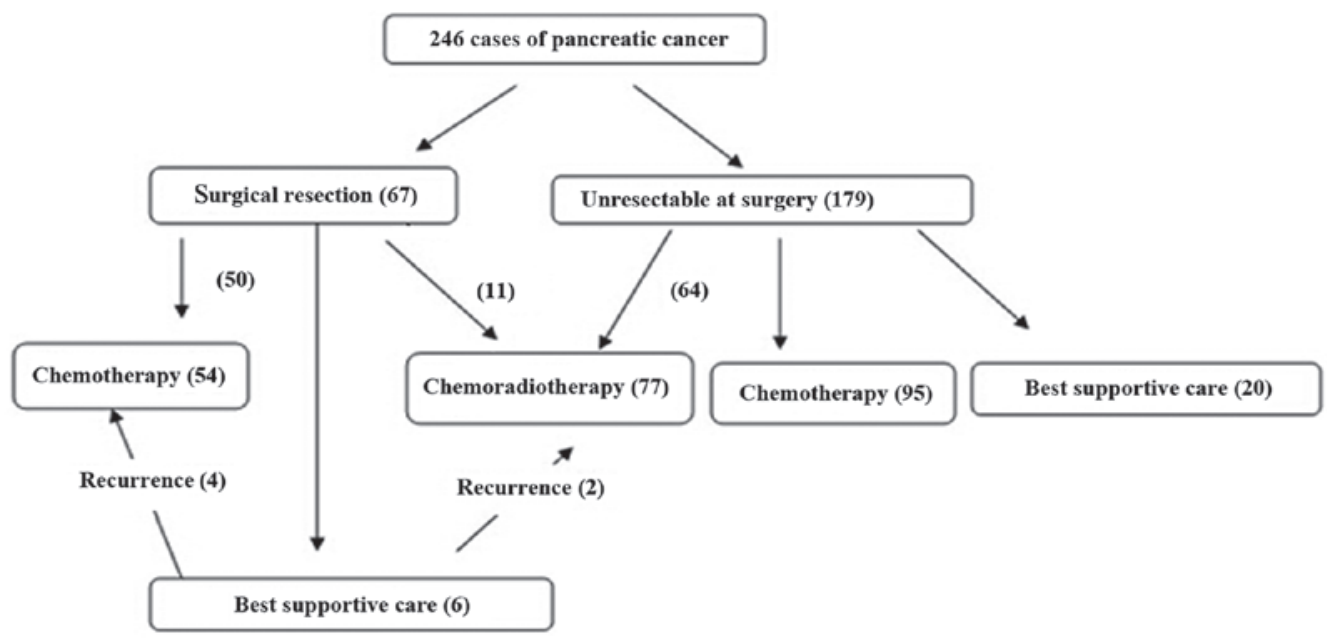

Figure 1. Flow chart of the treatment of all the pancreatic cancer patients.

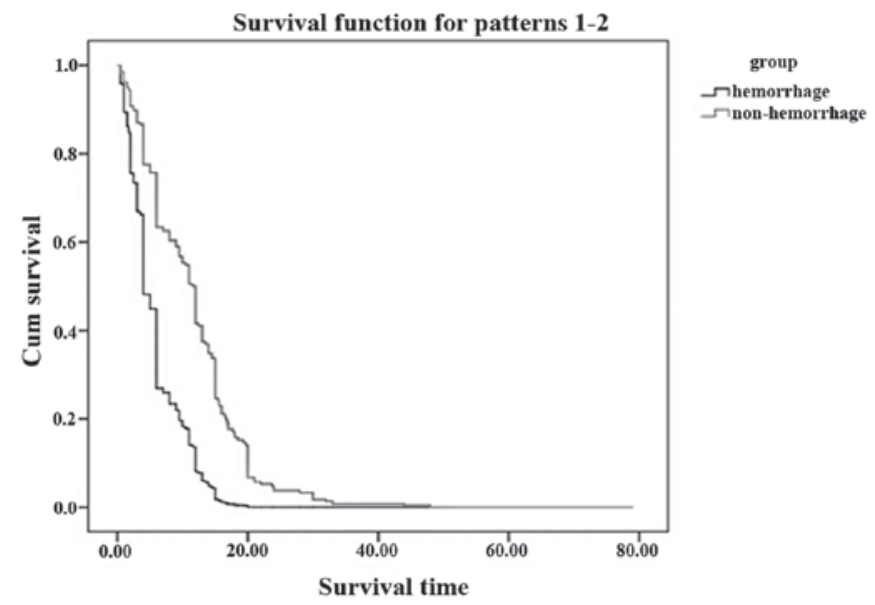

Figure 2. Comparision of the survival time between the gastrointestinal (GI) hemorrhage patients and non-GI hemorrhage patients. The overall median survival time was 14.5 months in the non-GI hemorrhage group and 9.0 months in the GI hemorrhage group.
Survival. A total of 212 patients $(86.2 \%)$ had succumbed at the time of final analysis. The median overall survival time was 9.0 months (range, 2.0-6.0 months) in patients without GI hemorrhage and 14.5 months (range, 0.5-48.0 months) in patients with GI hemorrhage (Fig. 2), although the difference of the overall survival time was significant with $\mathrm{P}<0.05$. The median period from clinically diagnosed GI hemorrhage to fatality was extremely short (30 days range from $1 \mathrm{~h}$ to 65 days).

\section{Discussion}

In certain cases, GI hemorrhage may be a symptom of a serious or life-threatening condition that should be immediately evaluated in an emergency setting. The present analysis showed that the median period from GI hemorrhage to mortality is extremely short for the patients with pancreatic cancer, and the median overall survival time of the patients 
Table V. Cox regression analysis of the effect of GI hemorrhage on the survival rate.

\begin{tabular}{|c|c|c|c|}
\hline Variable & P-value & HR & $95.0 \% \mathrm{CI}$ for $\mathrm{HR}$ \\
\hline Age $>65$ years & 0.968 & 1.000 & $0.985-1.016$ \\
\hline Gender, male & 0.125 & 1.352 & $0.919-1.989$ \\
\hline Alcohol, yes/no & 0.929 & 1.021 & $0.641-1.626$ \\
\hline Smoking, yes/no & 0.271 & 0.801 & $0.540-1.189$ \\
\hline Past hemorrhage history, yes/no & 0.819 & 1.095 & $0.503-2.384$ \\
\hline Past ulcer history, yes/no & 0.142 & 0.458 & $0.162-1.298$ \\
\hline Past diabetes history, yes/no & 0.440 & 0.808 & $0.470-1.388$ \\
\hline Past cirrhosis history, yes/no & 0.487 & 0.350 & $0.540-1.650$ \\
\hline Initial stage (I) & 0.056 & & \\
\hline Initial stage (II) & 0.017 & 0.540 & $0.326-0.895$ \\
\hline Initial stage (III) & 0.811 & 1.135 & $0.400-3.220$ \\
\hline Initial stage (IV) & 0.390 & 1.246 & $0.754-2.060$ \\
\hline Initial clinical symptoms (stained yellow) & 0.019 & & \\
\hline Initial clinical symptoms (abdominal distension or pain) & 0.050 & 2.117 & $0.999-4.490$ \\
\hline Initial clinical symptoms (diabete) & 0.017 & 2.299 & $1.162-4.550$ \\
\hline Initial clinical symptoms (ileus) & 0.001 & 4.552 & $1.874-11.060$ \\
\hline Initial clinical symptoms (others) & 0.095 & 2.173 & $0.873-5.408$ \\
\hline Tumor location (head of pancreas) & 0.225 & & \\
\hline Tumor location (body of pancreas) & 0.093 & 0.674 & $0.425-1.068$ \\
\hline Tumor location (tail of panceas) & 0.697 & 0.906 & $0.549-1.493$ \\
\hline CA19-9 & 0.668 & 0.924 & $0.646-1.324$ \\
\hline
\end{tabular}

GI, gastrointestinal; CI, confidence interval; CA19-9, carbohydrate antigen 19-9, HR, hazard ratio.

with GI hemorrhage was significantly shorter than that of patients without GI hemorrhage, which was similar with other studies (18-20), and is critical if not treated properly. The main treatment for GI hemorrhage in patients include fasting, proton pump inhibitor and endoscopic therapy, which may be effective to a certain degree, however, the effect to patients of GI hemorrhage with pancreatic cancer was not so clear, with an even shorten survival rate. The patients of pancreatic cancer are common in a significant tumor load state and their body condition is extremely poor, therefore, clinical doctors are usually unable to do anything. From this perspective, it is critical to early diagnose GI hemorrhage and to prevent it as soon as possible.

There are certain studies that are committed to the cause prevention and the treatment of GI hemorrhage, while there are few studies that focus on the early prevention, early diagnosis and the reasonable treatment of GI hemorrhage associated with pancreatic cancer. Regarding the risk factors for GI hemorrhage of pancreatic cancer, a study reported that the independent risk factor for GI hemorrhage was tumor location in the pancreatic body (21). The patients enrolled in the present study received CRT for pathologically proven LAPC. However, there are few studies that reported the risk factors of all the stages of pancreatic cancer patients. In the present study, the initial clinical tumor stage of IV was one of the main factors $(\mathrm{OR}, 21.94, \mathrm{P}=0.008)$. To a certain degree, the late stage of pancreatic cancer as the independent risk factor is understandable and it is likely associated with numerous factors, such as heavy tumor burden, complex treatment, poor physical fitness of patients and the larger mass may even increase the probability of violations of the digestive tract. Thus, more studies are required to investigate whether there is any difference between the different tumor stages or the different treatment.

The present study has several limitations. First, it was a retrospective study and a small number of patients were evaluated. Second, regarding the therapy of pancreatic cancer, the chemotherapy and pain management-induced adverse effects could not be excluded. In addition, the regimens were different between each other and the numbers of patients receiving gemcitabine differed from those receiving 5-FU, and several studies have reported that gemcitabine is more toxic than 5-FU $(22,23)$. Third is hospital stay and blood transfusion, which were evaluated in other studies $(24,25)$. Therefore, further studies are required to improve the outcomes in GI hemorrhage patients with pancreatic cancer.

In conclusion, the present results show that GI hemorrhage is critical in pancreatic cancer patients. Not only was the median overall survival rate of the patients with GI hemorrhage significantly shorter than that of patients without GI hemorrhage, but the time from GI hemorrhage to mortality was also extremely short. Clinical oncologists should pay more attention to those who had a late stage of pancreatic cancer. The clinicians should consider GI hemorrhage in pancreatic cancer patients. Extensive studies are required to explore more effective treatment measures. 


\section{References}

1. Jemal A, Siegel R, Ward E, Hao Y, Xu J and Thun MJ: Cancer statistics, 2009. CA Cancer J Clin 59: 225-249, 2009.

2. Olowokure O and Qi X: Pancreatic cancer: Current standards, working towards a new therapeutic approach. Expert Rev Anticancer Ther 14: 495-497, 2014.

3. Winek T, Hamre D, Mozell E and Vetto RM: Prognostic factors for survival after pancreaticoduodenectomy for malignan disease. Am J Surg 159: 454-456, 1990.

4. Rockey DC: To transfuse or not to transfuse in upper gastrointestinal hemorrhage? That is the question. Hepatology 60 422-424, 2014

5. Longstreth GF: Epidemiology and outcome of patients hospitalized with acute lower gastrointestinal hemorrhage: A population-based study. Am J Gastroenterol 92: 419-424, 1997

6. Longstreth GF: Epidemiology of hospitalization for acute upper gastrointestinal hemorrhage: A population-based study. Am J Gastroenterol 90: 206-210, 1995.

7. Wollenman CS, Chason R, Reisch JS and Rockey DC: Impact of ethnicity in upper gastrointestinal hemorrhage. J Clin Gastroenterol 48: 343-350, 2014.

8. Fallah MA, Prakash C and Edmundowicz S: Acute gastrointestinal bleeding. Med Clin North Am 84: 1183-1208, 2000.

9. Greenspoon J, Barkun A, Bardou M, Chiba N, Leontiadis GI, Marshall JK, Metz DC, Romagnuolo J and Sung J; International Consensus Upper Gastrointestinal Bleeding Conference Group: Management of patients with nonvariceal upper gastrointestinal bleeding. Clin Gastroenterol Hepatol 10: 234-239, 2012.

10. Cooper GS, Chak A, Way LE, Hammar PJ, Harper DL and Rosenthal GE: Early endoscopy in upper gastrointestinal hemorrhage: Associations with recurrent bleeding, surgery and length of hospital stay. Gastrointest Endosc 49: 145-152, 1999.

11. Simoens M, Gevers AM and Rutgeerts P: Endoscopic therapy for upper gastrointestinal hemorrhage: A state of the art. Hepatogastroenterology 46: 737-745, 1999

12. Longstreth GF and Feitelberg SP: Outpatient care of selected patients with acute non-variceal upper gastrointestinal haemorrhage. Lancet 345: 108-111, 1995.

13. Silverstein FE, Gilbert DA, Tedesco FJ, Buenger NK and Persing J: The national ASGE survey on upper gastrointestinal bleeding. II. Clinical prognostic factors. Gastrointest Endosc 27: 80-93, 1981.

14. Laine L, Yang H, Chang SC and Datto C: Trends for incidence of hospitalization and death due to GI complications in the United States from 2001 to 2009. Am J Gastroenterol 107: 1190-1195, quiz 1196, 2012.
15. Charbonnet P, Toman J, Bühler L, Vermeulen B, Morel P, Becker CD and Terrier F: Treatment of gastrointestinal hemorrhage. Abdom Imaging 30: 719-726, 2005.

16. Bone RC, Balk RA, Cerra FB, Dellinger RP, Fein AM, Knaus WA, Schein RM and Sibbald WJ; The ACCP/SCCM Consensus Conference Committee. American College of Chest Physicians/Society of Critical Care Medicine: Definitions for sepsis and organ failure and guidelines for the use of innovative therapies in sepsis. Chest 101: 1644-1655, 1992.

17. Davenport RJ, Dennis MS and Warlow CP: Gastrointestinal hemorrhage after acute stroke. Stroke 27: 421-424, 1996.

18. Shinchi H, Takao S, Noma H, Matsuo Y, Mataki Y, Mori S and Aikou T: Length and quality of survival after external-beam radiotherapy with concurrent continuous 5-fluorouracil infusion for locally unresectable pancreatic cancer. Int J Radiat Oncol Biol Phys 53: 146-150, 2002.

19. Li CP, Chao Y, Chi KH, Chan WK, Teng HC, Lee RC, Chang FY, Lee SD and Yen SH: Concurrent chemoradiotherapy treatment of locally advanced pancreatic cancer: Gemcitabine versus 5-fluorouracil, a randomized controlled study. Int J Radiat Oncol Biol Phys 57: 98-104, 2003.

20. Chauffert B, Mornex F, Bonnetain F, Rougier P, Mariette C, Bouché O, Bosset JF, Aparicio T, Mineur L, Azzedine A, et al: Phase III trial comparing intensive induction chemoradiotherapy (60 Gy, infusional 5-FU and intermittent cisplatin) followed by maintenance gemcitabine with gemcitabine alone for locally advanced unresectable pancreatic cancer. Definitive results of the 2000-01 FFCD/SFRO study. Ann Oncol 19: 1592-1599, 2008.

21. Lee KJ, Kim HM, Jung JW, Chung MJ, Park JY, Bang S, Park SW, Lee WJ, Seong JS and Song SY: Gastrointestinal hemorrhage after concurrent chemoradiotherapy in locally advanced pancreatic cancer. Gut Liver 7: 106-111, 2013.

22. Crane CH, Abbruzzese JL, Evans DB, Wolff RA, Ballo MT, Delclos M, Milas L, Mason K, Charnsangavej C, Pisters PW, et al: Is the therapeutic index better with gemcitabine-based chemoradiation than with 5-fluorouracil-based chemoradiation in locally advanced pancreatic cancer? Int J Radiat Oncol Biol Phys 52: 1293-1302, 2002

23. Huguet F, Girard N, Guerche CS, Hennequin C, Mornex F and Azria D: Chemoradiotherapy in the management of locally advanced pancreatic carcinoma: A qualitative systematic review. J Clin Oncol 27: 2269-2277, 2009.

24. Cheung J, Yu A, LaBossiere J, Zhu Q and Fedorak RN: Peptic ulcer bleeding outcomes adversely affected by end-stage renal disease. Gastrointest Endosc 71: 44-49, 2010.

25. Toke AB: GI bleeding risk in patients undergoing dialysis. Gastrointest Endosc 71: 50-52, 2010. 